\title{
Enfermedad de Niemann Pick tipo-A. Presentación de 12 casos
}

\author{
Zarco-Román J+1, Romero-Gómez $\mathrm{HE}^{2}$, Carbajal-Rodríguez $\mathrm{L}^{3}$
}

\begin{abstract}
Resumen
INTRODUCCIÓN: la enfermedad de Niemann Pick tipo A es una enfermedad hereditaria de depósito lisosomal, debida a un déficit de la enzima esfingomielinasa ácida, lo que da lugar a la acumulación de esfingomielina en las células del sistema reticuloendotelial y nervioso central.
\end{abstract}

OBJETIVO: se describe el curso de la enfermedad de Niemann Pick tipo A en 12 pacientes: tres niños y nueve niñas, con edades entre 3 a 33 meses.

MATERIAL Y MÉTODOS: estudio retrospectivo efectuado con base en la información de los expedientes de 12 pacientes, evaluados mediante un examen físico completo, oftalmológico, análisis de laboratorio, ultrasonido de abdomen, tomografía axial computarizada o resonancia magnética nuclear cerebral, radiografías de tórax, ecocardiograma, electroencefalograma, potenciales evocados auditivos y visuales, serie esofagogastroduodenal y serie ósea. Se obtuvo información sobre enfermedades intercurrentes y causas de muerte.

RESULTADOS: la sintomatología comenzó en promedio a los 5.9 meses (2 a 16 meses) y el diagnóstico definitivo se realizó a la edad de los 15.6 meses ( 3 a 33 meses) a través del cuadro clínico sugestivo, con presencia de células espumosas en el aspirado de médula ósea 12/12 (100\%) y deficiencia de la enzima esfingomielinasa ácida en $12 / 12$ pacientes $(100 \%)$; la biopsia hepática fue sugestiva de enfermedad por atesoramiento en 5/12 (41.6\%). Las manifestaciones clínicas por orden de frecuencia fueron: hepatoesplenomegalia y afección neurológica en 12/12 (100\%), alteración pulmonar 11/12 (91.6\%), hematológica 9/12 (80\%), oftalmológica con mancha rojo cereza 9/12 (75\%), ósea $6 / 12(50 \%)$, y cardiaca $4 / 12(33.3 \%)$. Únicamente hubo antecedente de consanguinidad en 4 familias (33.3\%).

CONCLUSIONES: el curso clínico de la enfermedad de Niemann Pick tipo A es muy similar entre los pacientes afectados, el primer dato clínico detectado es la hepatoesplenomegalia. Los pacientes están asintomáticos los primeros 4 meses de vida, posterior a los cuales presentan un curso neurodegenerativo que conduce a la muerte antes de los 3 años.

PALABRAS CLAVE: enfermedad de Niemann Pick tipo A, mancha rojo cereza, hepatoesplenomegalia.
${ }^{1}$ Médico adscrito al servicio de Medicina Interna. ${ }^{2}$ Médico pediatra, ex-residente.

${ }^{3}$ Jefe del servicio de Medicina Interna.

Instituto Nacional de Pediatría, México.

Recibido: 29 de abril del 2015

Aceptado: 7 de octubre del 2016

Correspondencia

Dr. Luis Carbajal Rodríguez

drcarbajalr@gmail.com

Este artículo debe citarse como

Zarco-Román J, Romero-Gómez HE, CarbajalRodríguez L. Enfermedad de Niemann Pick tipoA. Presentación de 12 casos. Acta Pediatr Mex. 2017;38(3):152-164. 


\section{Niemann Pick disease type-A: presentation of 12 cases.}

\author{
Zarco-Román J+1, Romero-Gómez HE², Carbajal-Rodríguez L³
}

\begin{abstract}
INTRODUCTION: Niemann Pick disease type A (ENP-A) is a lysosomal storage disease caused by a deficit of acid sphingomyelinase enzyme (ASM), which results in the accumulation of sphingomyelin in cells of the reticuloendothelial and central nervous system.
\end{abstract}

OBJECTIVE: We describe the clinical course of the ENP-A in twelve patients, three boys and nine girls, between the ages of 3-33 months.

MATERIAL AND METHODS: The retrospective study was based on the medical files of the 12 patients, evaluated with a complete physical and ophthalmologic examination, as well as laboratory tests, abdominal ultrasound, computed tomography $(\mathrm{CT})$ or magnetic resonance imaging (MRI) of the brain, chest radiography, echocardiography, EEG, upper gastrointestinal series, and bone age. Information about intercurrent diseases and causes of death, were obtained.

RESULTS: The symptoms started at 5.9 months (2-16 months) and the definitive diagnosis was made at the age of 15.6 months (3-33 months) through suggestive clinical manifestations, with a presence of foam cells in the bone marrow of $12 / 12$ patients (100\%) and ASM enzyme deficiency in $12 / 12$ patients $(100 \%)$; hepatic biopsy was suggestive in 5/12 (41.6\%). Clinical signs in order of frequency were: hepatosplenomegaly and neurological disease in $12 / 12(100 \%)$, lung disorder $11 / 12(91.6 \%)$, hematologic affection $9 / 12(80 \%)$, cherry-red spot $9 / 12$ (75\%), bone involved 6/12 (50\%), and cardiac disease 4/12 $(33.3 \%)$. Only 4 cases $(33.3 \%)$ were consanguineous.

CONCLUSIONS: The clinical course of the ENP-A is very similar among affected patients; the first clinical manifestation detected was hepatosplenomegaly. The first 4 months of life of the patients were asymptomatic; then they showed a neurodegenerative course that leads to death before reaching the age of 3 years.

KEYWORDS: Niemann Pick type A; cherry-red spot; hepatosplenomegaly

\footnotetext{
${ }^{1}$ Médico adscrito al servicio de Medicina Interna.

${ }^{2}$ Médico pediatra, ex-residente.

3Jefe del servicio de Medicina Interna.
}

Instituto Nacional de Pediatría, México.

Correspondence

Dr. Luis Carbajal Rodríguez

drcarbajalr@gmail.com

\section{INTRODUCCIÓN}

La enfermedad de Niemann Pick tipo A es una enfermedad hereditaria de depósito lisosomal, debida a un déficit de la enzima esfingomielinasa ácida, codificada por el gen SMPD1 localizado en el cromosoma 11p15.1-p15.4, lo que da lugar a la acumulación de esfingomielina (un 
esfingolípido) en las células del sistema monocito-macrófago, especialmente de las células y del sistema reticuloendotelial y nervioso. ${ }^{1}$

La enfermedad de Niemann Pick debe su nombre a la primera descripción clínica en 1914 por el pediatra Albert Niemann e histológica en 1927 por el patólogo Ludwig Pick. ${ }^{2,3}$ Posteriormente en 1961 y 1966, los trabajos por separado de Allen Crocker y Roscoe Brady permitieron conocer más acerca de los sustratos bioquímicos de la enfermedad y generar una clasificación en cuatro tipos: A, B, C y D. ${ }^{4}$

La enfermedad de Niemann Pick tipo A se presenta en todas las razas, la prevalencia global es de 1:250,000 recién nacidos vivos, aunque la incidencia es mayor entre los judíos Ashkenazi $(1: 40,000)$, pues su frecuencia de portadores es de 1:100 y de éstos $90 \%$ de los casos son debidos a 3 mutaciones (p.Arg496Leu, p.Leu302Pro y c.996delC (fsPro330)) en el gen SMPD1. ${ }^{5,6}$ Su patrón de herencia es autosómica recesiva. No hay datos en relación a su incidencia en México; sin embargo, en Latinoamérica se ha descrito que en Chile la mutación p.Ala359Asp tiene una frecuencia de portadores de 1:105, ${ }^{6}$ prediciendo con ello una incidencia de la enfermedad en 1:44,960. ${ }^{7}$

La enfermedad tipo A es la forma más frecuente de enfermedad de Niemann Pick ya que está presente hasta en $75 \%$ de los casos. Los pacientes afectados tienen hepatoesplenomegalia severa que se detecta durante los primeros cuatro meses de vida. La alteración neurológica ocurre como un deterioro temprano de la función motora en los primeros meses de vida, caracterizada por regresión del neurodesarrollo. La mayoría llegan a sentarse y pocos a gatear. La pérdida rápida y progresiva de la función neurológica global conduce a la muerte en los primeros 2 a 3 años de vida. Tienen falla para crecer pues los trastornos en la succión-deglución dificultan la alimentación, lo cual favorece las microaspiraciones. ${ }^{8}$
El 50\% de los casos tienen mancha rojo cereza a nivel del fondo de ojo al momento del diagnóstico y casi todos los pacientes la desarrollan durante la progresión de la enfermedad. ${ }^{9} \mathrm{Se}$ observan grandes concentraciones de células espumosas en el bazo, hígado y médula ósea, cuya presencia es sugestiva de la enfermedad, pero no diagnóstica. Para confirmar el diagnóstico de los tipos A y B se requiere la demostración del déficit de la enzima esfingomielinasa ácida en leucocitos o fibroblastos. ${ }^{10}$

También el análisis molecular del gen SMPD1 puede confirmar el diagnóstico. La tasa de detección de mutaciones a través de la secuenciación es de $\sim 95 \% .{ }^{11}$ Se han reportado más de 100 variantes patogénicas; sin embargo, sólo algunas presentan correlación con el fenotipo ${ }^{12,13}$ y otras, como la mutación p.Trp391Gly, tienen un amplio espectro de manifestaciones, que van desde enfermedad retinal subclínica hasta ataxia severa, déficits cognitivos y desordenes psiquiátricos. ${ }^{14}$ La mutación p.Arg610del está asociada a un fenotipo leve de la enfermedad tipo B, y fue considerada como neuroprotectora; $\sin$ embargo, se ha reportado en casos que muestran diversos cuadros clínicos, por lo que no guarda una clara correlación genotipo-fenotipo, sugiriendo la presencia de otros factores que modifican la presentación clínica en los afectados. ${ }^{15}$

No existe un tratamiento específico para esta enfermedad. Se utilizan medidas como fórmulas altas en calorías, instauración de sondas nasogástricas o de gastrostomía, uso temprano de antibióticos y fisioterapia pulmonar, medidas de confort, procinéticos, anticonvulsivos, rehabilitación y medidas de soporte invasivas, cuando los familiares así lo decidan, y siempre enterados del pronóstico desfavorable para la vida de este tipo de pacientes. Hay terapias experimentales como el trasplante de médula ósea, que no ha mostrado un beneficio en la evolución neurológica, aunque hay casos reportados en donde 
hubo mejoría de la visceromegalia, la afección pulmonar y la presencia de células espumosas en pacientes con enfermedad de Niemann Pick tipo B. ${ }^{16} \mathrm{El}$ trasplante hepático en un paciente con enfermedad de Niemann Pick tipo A ha presentado un beneficio terapéutico transitorio. ${ }^{17}$ En ambos tipos la esfingomielinasa ácida es deficiente. La enfermedad tipo B tiene una aparición más tardía durante la infancia o la adolescencia y es menos severa que la tipo A con alta supervivencia en la edad adulta, ya que la actividad de la esfingomielinasa es mayor. Se ha analizado la actividad residual en fibroblastos cultivados siendo de 1 y $4 \%$ en A y B, respectivamente. ${ }^{18}$ Cursan con la misma afectación visceral, pero la mayoría de los pacientes con enfermedad tipo B no tiene alteración neurológica, aunque al final de la primera infancia algunos pacientes cursan con diversos grados de afectación del sistema nervioso central. ${ }^{19} \mathrm{~A}$ diferencia de lo que ocurre en la enfermedad de Niemann Pick tipo C, donde existe un defecto en el transporte intracelular de colesterol y otros glicoesfingolípidos, y las mutaciones se encuentran en los genes NPC1 y $N P C 2 .{ }^{20}$ El diagnóstico de la enfermedad tipo C a menudo se retrasa debido al amplio espectro de fenotipos clínicos. Las manifestaciones sistémicas están presentes y siempre preceden a la aparición de las manifestaciones neurológicas. La mayoría de los signos neurológicos comunes son la parálisis vertical supranuclear, ataxia cerebelosa, disartria, disfagia y la demencia progresiva. La cataplejía, convulsiones y distonía son otras características comunes. En 95\% de las familias se encuentran mutaciones en el gen NPC1 y el resto en NPC2. ${ }^{21}$

Los pacientes con enfermedad de Niemann Pick tipo D comparten manifestaciones clínicas similares con los pacientes con enfermedad tipo C. Se caracterizan por un compromiso subagudo del sistema nervioso, un curso moderado y lento de la enfermedad, así como menor almacenamiento visceral. En un estudio de Nueva Escocia, los pacientes descritos con enfermedad tipo D compartían un ancestro en común, y actualmente con el mejor entendimiento de la genética se sabe que presentaban una mutación específica en el gen NPC1, por lo cual estos pacientes en realidad tenían una enfermedad tipo C..$^{21-22}$

A través de modelos en ratón se ha estudiado y caracterizado la neuropatología asociada con la deficiencia de esfingomielinasa ácida, lo que también ha permitido evaluar diversas intervenciones terapéuticas ${ }^{23,24}$ como la infusión intracerebroventricular de esfingomielinasa ácida humana purificada que ha demostrado una mejoría y un alivio parcial de las anormalidades motoras en estos ratones. ${ }^{25}$

También se ha evaluado el trasplante intracerebral de células progenitoras neuronales, que expresan esfingomielinasa ácida humana (hASM) en ratones con enfermedad tipo A in vitro, sobreviviendo hasta 10 semanas. In vivo apenas expresaron hASM a niveles detectables pero suficientes para revertir la patología lisosomal. ${ }^{26}$ De igual manera, destaca la transferencia de genes mediados por vectores, lo cuales in vitro incrementaron 16 veces los valores de hASM en fibroblastos ${ }^{27} \mathrm{e}$ in vivo inyectados en cerebelo han logrado expresar hASM a lo largo del cerebelo, tallo, mesencéfalo y médula espinal. Lo que apoya el desarrollo de terapia génica en la enfermedad de Niemann Pick tipo A. ${ }^{28}$ Actualmente, se estudia la terapia de reemplazo enzimático con esfingomielinasa recombinante como tratamiento potencial para la enfermedad tipo B. ${ }^{29}$

\section{MATERIAL Y MÉTODOS}

Se realizó un estudio retrospectivo con base en la información de los expedientes de 12 pacientes con enfermedad de Niemann Pick tipo A (9 femeninos, 3 masculinos), diagnosticados en el departamento de Medicina Interna del Instituto 
Nacional de Pediatría durante el periodo del 2000 al 2013. Se describió la evolución clínica y los datos obtenidos en el examen oftalmológico, medidas antropométricas: peso, talla y perímetro cefálico; análisis de laboratorio, ultrasonido de abdomen, tomografía de cráneo o resonancia magnética cerebral, radiografías de tórax, serie ósea, ecocardiograma, electroencefalograma, potenciales auditivos y visuales, serie esofagogastroduodenal, estudios de histopatología y se obtuvo información sobre enfermedades intercurrentes y causas de la muerte.

\section{RESULTADOS}

Los síntomas iniciaron en promedio a los 5.9 meses (2-16 meses), fueron referidos de otras instituciones a la edad promedio de 13.4 meses (8-33 meses) con edad al diagnóstico en promedio de 15.6 meses (3 a 33 meses) (Cuadro 1). Cuatro de 12 familias contaban con consanguinidad en tercer grado. Un paciente tenía antecedente de 2 hermanos fallecidos a los 2 y 4 años con cuadro clínico compatible con enfermedad de Niemann Pick tipo A, siendo el único caso de más de un paciente afectado en la familia. Sin embargo, el diagnóstico enzimático no fue realizado, ya que no fueron atendidos en nuestro hospital.

Cuadro 1. Curso de la enfermedad del grupo de pacientes con enfermedad de Niemann Pick tipo A

\begin{tabular}{|l|c|c|}
\hline \multirow{2}{*}{} & \multicolumn{2}{|c|}{ Edad en meses } \\
\cline { 2 - 3 } & Promedio & Min-Máx \\
\hline Primer síntoma & 5.9 & $2-16$ \\
\hline Diagnóstico & 15.6 & $3-33$ \\
\hline Signos neurológicos & 5.5 & $3-7$ \\
\hline Síntomas gastrointestinales & 10.2 & $2-24$ \\
\hline $\begin{array}{l}\text { Problemas a la alimentación } \\
\text { y deglución }\end{array}$ & 8.2 & $6-19$ \\
\hline Falla del medro & 6.7 & $3-12$ \\
\hline Síntomas respiratorios & 11.1 & $3-19$ \\
\hline Irritabilidad & 13 & $6-19$ \\
\hline Muerte & 24 & $20-28$ \\
\hline
\end{tabular}

La evolución del desarrollo psicomotor de los pacientes fue: todos fijaron la mirada en promedio a los 3 meses y la perdieron a los 17.5 meses; la sonrisa social apareció en promedio a los 2.4 meses y se perdió a los 15.1 meses; el seguimiento visual a los 4 meses y se perdió a los 11 meses; el sostén cefálico a los 3.7 meses de edad y se perdió en promedio a los 12.5 meses; balbuceo a los 8.6 meses y se perdió a los 17.8 meses. Solamente 3/12 lograron la sedestación con apoyo y uno de éstos logró la sedestación de manera independiente. Ninguno gateó, ni logró la bipedestación o caminó. Se reporta transferencia de objetos en 4/12 con inicio promedio a los 10 meses y pérdida en todos a los 20.6 meses. Todos mantuvieron interacción con sus padres hasta la edad de 15.1 meses (Cuadro 2). 9/12 pacientes presentaron hipotonía en la primera evaluación, posteriormente desarrollaron espasticidad de extremidades inferiores, temblor distal e hiperreflexia. Un paciente presentó nistagmo. Todos recibieron terapia de rehabilitación y tuvieron durante la evolución algún grado de desnutrición.

Al ingreso, únicamente cuatro pacientes presentaban crisis convulsivas, sin embargo, en su evolución todos las desarrollaron, e inclusive un paciente ingresó a urgencias por descontrol de crisis convulsivas. 9/12 pacientes tuvieron la mancha rojo cereza, promedio a los 13.2 meses;

Cuadro 2. Obtención y pérdida (en meses) de los hitos del desarrollo en el grupo de pacientes con enfermedad de Niemann Pick tipo A

\begin{tabular}{|l|c|c|}
\hline & Obtención & Pérdida \\
\hline Fija la mirada & 3 & 11 \\
\hline Sigue con la mirada & 4 & 17.5 \\
\hline Sonrisa refleja & 2.4 & 15.1 \\
\hline Sostén cefálico & 3.7 & 12.4 \\
\hline Sedestación sin apoyo & 8 & 18 \\
\hline Balbuceo & 8.6 & 17.8 \\
\hline Transferencia de objetos & 10 & 20.6
\end{tabular}


los problemas gastrointestinales aparecieron a la edad promedio de 8.2 meses y fueron constantes en 11/12 pacientes. En 3/11 el vómito fue frecuente, en 6/11 diarrea que alternaba con periodos de estreñimiento, en 3/11 intolerancia alimentaria caracterizada por periodos de distensión abdominal, y 10/12 presentaron alteración en la deglución.

En 11/12 pacientes se instauraron medidas para asegurar la dieta, como sonda nasogástrica o transpilórica y posteriormente se les realizó gastrostomía. Un paciente de tres meses mantiene aún alimentación por succión.

La visceromegalia fue el primer síntoma, presente en 12/12, corroborada por ultrasonido abdominal y se detectó en promedio a la edad de 5.9 meses, (2-16 meses). Hepatomegalia en todos los pacientes con una media al ingreso de $6.4 \mathrm{~cm}$ debajo del borde costal derecho, progresando y llegando a ser masiva en 2 de ellos

(Figura 1). Esplenomegalia en 9/12 pacientes al inicio, con una media de $8 \mathrm{~cm}$ debajo del borde costal izquierdo, que progresó y llegó a ser masiva en 4 de ellos. Hubo ascitis en 3/12 pacientes, en promedio a los 20 meses de edad. Dos pacientes cursaron con ictericia

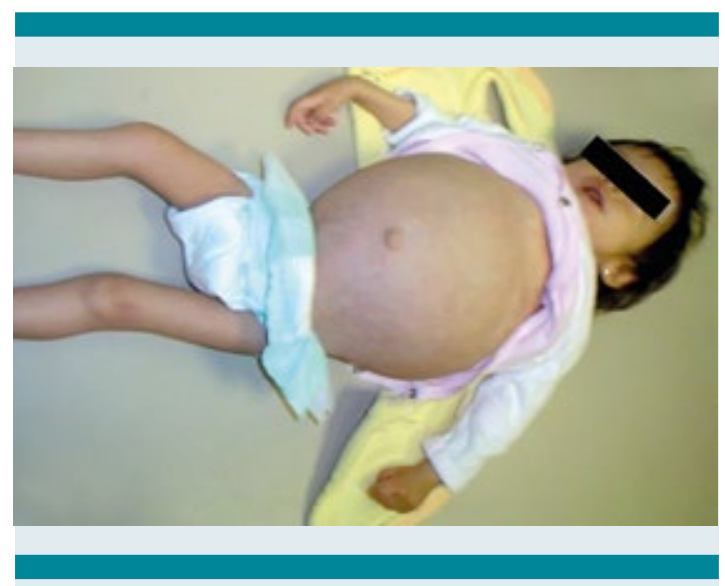

Figura 1. Hepatoesplenomegalia masiva en un paciente con enfermedad de Niemann Pick tipo A. desde el primer día de vida, que remitió hasta el sexto mes.

La afección pulmonar se evidenció en 11/12 pacientes, en 8/12 pacientes la radiografía de tórax presentó un patrón de infiltración intersticial retículo nodular en la primera evaluación (Figura 2). Se documentan 15 hospitalizaciones por neumonía, dos pacientes requirieron ventilación mecánica debido al deterioro ventilatorio y saturación de oxigeno menor a $80 \%$.

La evaluación cardiaca fue anormal en 4/12. Se realizó ecocardiograma demostrando foramen oval en 3 pacientes y en uno insuficiencia tricuspídea. En uno de ellos hubo disfunción miocárdica septal y disfunción leve del ventrículo izquierdo.

Se efectuó tomografía o resonancia magnética cerebral en todos los casos, la mayoría con atrofia corticosubcortical y retraso de la mielinización. En uno se reportó un hematoma subaracnoideo frontotemporoparietal izquierdo y en otro quistes aracnoideos en región temporal.

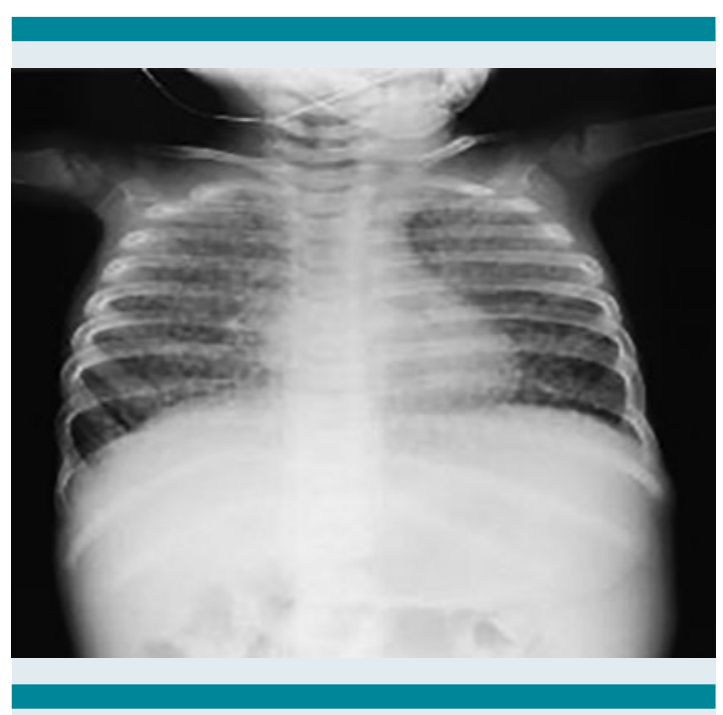

Figura 2. Radiografía de tórax en un paciente con enfermedad de Niemann Pick tipo A, obsérvese el patrón micronodular difuso. 
Se realizaron potenciales auditivos evocados a 9/12 pacientes con resultados anormales, 6/9 con hipoacusia profunda de manera bilateral. Potenciales visuales en 9/12, reportando 4 de ellos con alteración en la dispersión cortical. Se realizó electroencefalograma en 9/12 pacientes, reportando lentificación generalizada y actividad paroxística en todos.

En la serie ósea encontramos adelgazamiento de la cortical, aumento de tamaño de metáfisis distales en fémur, tibia y radios y desmineralización ósea en 6/12 pacientes.

Los niveles séricos de aspartatoaminotrasnferasa y alaninoaminotrasnferasa se reportaron elevados en todos los pacientes desde su primera evaluación. La bilirrubina total y la bilirrubina directa también tuvieron un incremento leve en 6 pacientes. El colesterol total se reportó incrementado en $4 / 12$ y los triglicéridos en 9/12 pacientes.

Las alteraciones hematológicas en 9/12: anemia en $7 / 12$, con hemoglobina menor a $9 \mathrm{mg} / \mathrm{dL}$ en 4 de ellos y plaquetopenia en 8/12 manifestando clínicamente petequias así como equimosis y un paciente con leucopenia (Cuadro 3).

Todos presentaron células espumosas en el aspirado de médula ósea, las células que se observan son de gran tamaño con núcleo excéntrico finamente vacuolado y de aspecto espumoso. Con la tinción de Giemsa algunos de los macrófagos pueden contener gránulos en el citoplasma de color azul verdoso que les confiere la denominación de histiocito azul marino.

En 5/12 pacientes se realizó biopsia hepática, encontrando acúmulo de material vacuolado sugestivo de enfermedad por atesoramiento. Se determinó la enzima esfingomielinasa ácida en leucocitos periféricos encontrándose deficiente en todos con lo que se corroboró el diagnóstico (valor de referencia 200-3500 pmol/spot*20 h).
Cuadro 3. Valores promedio de laboratorio al inicio y final de la evaluación

\begin{tabular}{l|c|c|}
\hline & $\begin{array}{c}\text { Al inicio de la } \\
\text { evaluación } \\
\text { (mín-máx) }\end{array}$ & $\begin{array}{c}\text { Al final de la } \\
\text { evaluación } \\
\text { (mín-máx) }\end{array}$ \\
\hline $\mathrm{Hb}, \mathrm{g} / \mathrm{dL}$ & $11.5(8.8-14.4)$ & $10.3(7.6-13.5)$ \\
\hline $\begin{array}{l}\text { Leucocitos/ } \\
\mathrm{mm} 3\end{array}$ & $9566(5300-17700)$ & $6412(1400-16900)$ \\
\hline $\begin{array}{l}\text { Plaquetas, } \\
\mathrm{x} 103 / \mathrm{mcL}\end{array}$ & $164(48-319)$ & $122(44-288)$ \\
\hline $\mathrm{BD}, \mathrm{mg} / \mathrm{dL}$ & $0.6(0.04-2.4)$ & $0.39(0.04-1.17)$ \\
\hline $\mathrm{BT}, \mathrm{mg} / \mathrm{dL}$ & $1.2(0.33-4.1)$ & $0.97(0.24-2.05)$ \\
\hline $\mathrm{CT}, \mathrm{mg} / \mathrm{dL}$ & $171.5(113-281)$ & $158.8(67-293)$ \\
\hline $\mathrm{TG}$ & $317.6(101-611)$ & $278(89-598)$ \\
\hline AST & $156.3(10-399)$ & $108.6(53-563)$ \\
\hline ALT & $270.7(9-1267)$ & $179.2(17-185)$ \\
\hline
\end{tabular}

ASP: aspartatoaminotrasnferasa; ALT: alaninoaminotrasnferasa: BD: bilirrubina directa; BT: bilirrubina total ; CT: colesterol total; $\mathrm{Hb}$ : hemoglobina; TG: triglicéridos.

Actualmente, están en seguimiento por la consulta externa 2 pacientes, los otros 10 han fallecido. Las causas de muerte intrahospitalaria fueron: falla hepática en uno, insuficiencia cardiaca descompensada en otro y un tercero con sepsis de foco pulmonar e insuficiencia respiratoria en quien sus familiares declinaron el uso de ventilación mecánica. Los otros 7 pacientes fallecieron en su domicilio. El promedio de tiempo del diagnóstico a la muerte fue de 11 meses, no se reportan sobrevivientes más allá de los 3 años. Se describen las manifestaciones clínicas y hallazgos más frecuentes de laboratorio y gabinete en pacientes con enfermedad de Niemann Pick tipo A (Cuadro 4).

\section{DISCUSIÓN}

Describimos la evolución de 12 pacientes con enfermedad de Niemann Pick tipo A, lo cual aporta mayor conocimiento sobre de las características e historia natural de la enfermedad. Nuestros pacientes tienen una evolución similar con un cuadro neurodegenerativo progresivo que 
Ileva invariablemente a la muerte, no más allá de los 3 años, acompañado de visceromegalia como primer signo, presencia de mancha rojo cereza, dificultades en la alimentación, pérdida de las habilidades motrices de manera temprana a pesar de la terapia de rehabilitación y procedimientos empleados como lo reportado en la bibliografía.

De manera general, los pacientes tienen un periodo neonatal normal, aunque dos de nuestros casos presentaron ictericia prolongada hasta los 6 meses, de los cuales uno requirió manejo con fenobarbital. Lo anterior coincide con lo descrito en el estudio de McGovern y Aron ${ }^{1}$ de 10 pacientes con enfermedad de Niemann Pick tipo A, pues reportan un paciente con ictericia neonatal, la cual se resuelve sin tratamiento. En nuestros pacientes el primer dato clínico identificado fue la visceromegalia, en promedio a los 5.9 meses de edad, aunque McGovern y Aron $^{1}$ lo identificaron más temprano (entre los 2 y 4 meses).

La valoración del neurodesarrollo en nuestros pacientes, se realizó en cada consulta por diferentes examinadores encontrando una detención del desarrollo psicomotor a partir de los 4 meses, algunos lograron la sedestación, pero ninguno la bipedestación. Se constató perdida de las habilidades del neurodesarrollo con la evolución de la enfermedad, perdiendo la interacción con sus padres en promedio a los 15.1 meses, lo cual también está acorde con lo previamente reportado, ${ }^{1}$ ya que en promedio el desarrollo psicomotor de los pacientes no es mayor de 6 meses con pérdida de interacción social hacia los 19 meses.

Encontramos que la hipotonía progresa habitualmente a una espasticidad de extremidades inferiores con hiperreflexia. También que las alteraciones tanto en la resonancia y tomografía de cráneo, como en los potenciales auditivos y visuales son hallazgos constantes. Y que las crisis convulsivas están presentes en el $100 \%$ de los casos, lo cual no había sido previamente descrito.

La mancha rojo cereza es un estigma observado en la enfermedad de Niemann Pick tipo A; sin embargo, no es patonogmónico, ya que otras enfermedades de depósito lisosomal la pueden presentar (Farber, galactosialidosis, gangliosidosis GM1, Tay-Sachs y sialidosis). En nuestros pacientes, no se demostró en tres, probablemente al encontrarse en un estadio temprano de la enfermedad al momento de la valoración, siendo posible su desarrollo conforme exista progresión de la enfermedad (Figura 3) pues McGovern y Aron lo reportan en $100 \%$ de sus pacientes hacia el año de edad. ${ }^{1}$

El compromiso pulmonar se observó en 91.6\% (Cuadro 4) de los pacientes, presentando infiltración intersticial micronodular en las radiografías de tórax, incluso en periodos asintomáticos, a pesar de que la auscultación pulmonar en gran

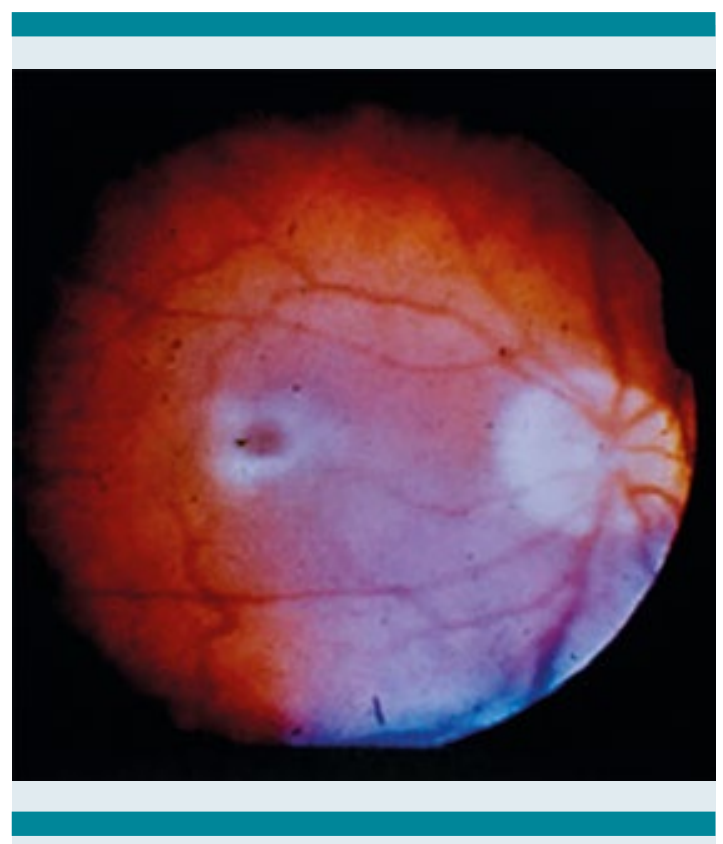

Figura 3. Fondo de ojo, mancha rojo cereza. 
Cuadro 4. Manifestaciones clínicas, hallazgos de laboratorio y gabinete en pacientes con enfermedad de Niemann Pick tipo A

\begin{tabular}{|l|c|}
\hline Hallazgos & $\%$ \\
\hline Hepatomegalia-esplenomegalia & 100 \\
\hline Detención de hitos del desarrollo & 100 \\
\hline Células espumosas en aspirado de médula ósea & 100 \\
\hline Enzima esfingomielinasa ácida en leucocitos & 100 \\
periféricos deficiente & 100 \\
\hline Falla de medro & 100 \\
\hline Crisis convulsivas & 100 \\
\hline AST y ALT incrementados & 91.60 \\
\hline Afectación pulmonar & 83.3 \\
\hline Dificultad para la deglución & 80.00 \\
\hline Alteraciones hematológicas (anemia o pla- & 75 \\
\hline quetopenia) & 75 \\
\hline Hipotonía generalizada & 66.6 \\
\hline Mancha rojo cereza & 58.3 \\
\hline Irritabilidad & 54.5 \\
\hline Espasticidad progresiva & 50 \\
\hline Diarrea/estreñimiento & 50 \\
\hline Neumonías de repetición & 33.3 \\
\hline Alteraciones óseas & 33.3 \\
\hline Alteraciones cardiacas & 33.3 \\
\hline Tórax restrictivo por visceromegalias & 25 \\
\hline Consanguinidad & 16.6 \\
\hline Enfermedad por reflujo gastroesofágico & 8.3 \\
\hline Ictericia neonatal prolongada & \\
\hline Nistagmo horizontal & 100 \\
\hline
\end{tabular}

ASP: aspartatoaminotrasnferasa; ALT: alaninoaminotrasnferasa.

parte de ellos fue normal; además, cursaron con infecciones de vías aéreas bajas de repetición. En un paciente la tomografía pulmonar constató enfisema panlobulillar, siendo dependiente de oxígeno desde los 8 meses. Un estudio realizado por Guillemot y sus colaboradores, evaluó el compromiso pulmonar en 13 niños con enfermedad de Niemann Pick tipos A ( $n=1), B(n=10)$ y C $(n=2)$, describen cómo los síntomas respiratorios estuvieron presentes al momento del diagnóstico en 10/13 y se desarrolló en todos a lo largo de su evolución, mostrando signos de afección pulmonar intersticial en las radiografías de tórax y tomografía pulmonar. En 7 pacientes el líquido de lavado broncoalveolar reveló acumulación de macrófagos espumosos. ${ }^{30}$ Es probable que el almacenamiento de esfingomielina en los macrófagos pulmonares conduzca a la aparición de enfermedad pulmonar intersticial, infecciones respiratorias frecuentes e insuficiencia respiratoria que es la causa de fallecimiento en la mayoría de los pacientes.

Hollak y sus colaboradores realizaron un reporte de 25 casos (21 pacientes con enfermedad de Niemann Pick tipo B y 4 con tipo A), si bien no describen la función pulmonar de los 4 pacientes con enfermedad tipo $A$, sí encuentran datos en 16 de 21 pacientes con enfermedad tipo $B, y$ describen diversos grados de enfermedad pulmonar restrictiva en 13 de ellos, con mayor afectación en las formas intermedias lo que sugiere una mayor acumulación de macrófagos espumosos a nivel pulmonar en relación con una menor actividad de la esfingomielinasa ácida. ${ }^{31}$

La hepatoesplenomegalia fue un dato constante y progresivo en nuestra serie, $100 \%$ con elevación de transaminasas y bilirrubinas, generando en algunos un incremento de la sintomatología respiratoria por un tórax restrictivo. Uno de los 3 pacientes con ascitis presentó falla hepática que condicionó su muerte en pocas semanas. Los 4 pacientes con enfermedad de Niemann Pick tipo A del reporte de Hollak y sus colaboradores cursaron con manifestaciones viscerales graves, incremento progresivo de los valores de las enzimas hepáticas. La ascitis fue también documentada en 2 pacientes por McGovern y Aron. ${ }^{1}$

En nuestra serie encontramos una mayor incidencia de defectos cardiacos, lo cual no es referido en la literatura internacional, inclusive la insuficiencia cardiaca fue una complicación que motivó el fallecimiento de uno de nuestros 
pacientes hospitalizados por lo que sugerimos que de manera rutinaria se realice valoración cardiológica en todos los pacientes con este diagnóstico. No se constató enfermedad ateroesclerótica a diferencia de un estudio de McGovern y sus colaboradores del 2004 donde se evaluaron a 10 pacientes con enfermedad de Niemann Pick tipo A y 30 con enfermedad tipo $B$, en el cual determinaron la lipoproteína de alta densidad (HDL) con valores anormales en el $100 \%$, (<35 mg/dL) así como hipertrigliceridemia e incremento de lipoproteína de baja densidad (LDL) en 62 y $67 \%$ respectivamente, lo cual se asocia a riesgo cardiovascular de ateroesclerosis de instauración temprana. ${ }^{32}$ En nuestra serie sólo encontramos incremento de colesterol y triglicéridos.

Los problemas para la deglución y demás síntomas gastrointestinales eventualmente llevan a la desnutrición a estos pacientes lo que indica la necesidad de la instauración de una sonda transpilórica o de gastrostomía de manera temprana, para facilitar la alimentación por parte del cuidador, asegurar un aporte nutricio adecuado y mejorar su calidad de vida. Otro beneficio probable sería la disminución de los eventos de broncoaspiración, pues al menos Salinas Velasco lo documentó en un caso con neumonía de repetición ${ }^{33}$ y McGovern y Aron reportan 24 hospitalizaciones por neumonía en 10 pacientes, ${ }^{1}$ y nosotros documentamos solo 15 hospitalizaciones por el mismo diagnóstico en 12 de nuestros pacientes.

La anemia y la plaquetopenia se hacen más acentuadas conforme progresa la enfermedad, lo cual derivó en la muerte por hemorragia en un paciente de la serie de McGovern y Aron ${ }^{1} y$ el desarrollo de un hematoma subdural en un caso del grupo de Hollak y sus colaboradores. ${ }^{31}$ En nuestros pacientes algunos tuvieron hemoglobina menor de 9, requiriendo transfusión de concentrados eritrocitarios, 3 pacientes con trombocitopenia menor de 50,000 requirieron transfusión de concentrados plaquetarios. Estos datos hematológicos son debidos por un lado a la infiltración de la médula ósea y por otro lado al hiperesplenismo que pueden presentar estos pacientes. De igual forma Salinas Velasco ${ }^{33}$ reporta en su paciente anemia con necesidad de transfusión de concentrado eritrocitario.

La terapia de rehabilitación inició desde el diagnóstico (edad promedio de 15.6 meses), pero el deterioro neurológico progresó, lo que motivó a la deserción por algunos cuidadores. Probablemente la instauración de una terapia temprana pudiera evidenciar mejores resultados, sin embargo, en uno de nuestros pacientes con diagnóstico a los 3 meses y referencia temprana a rehabilitación no se documentó mejoría en relación a la evolución de la enfermedad.

La mayoría de las familias eligieron no utilizar medidas de soporte invasivas, ya que habían recibido información y ayuda psicológica por el servicio de Cuidados Paliativos. Consecuentemente, estos pacientes fallecieron en su domicilio, recibiendo el aporte de dieta, líquidos, analgésicos y medicamentos administrados a través de sonda de gastrostomía. Las causas de muerte en las series comentadas describen principalmente insuficiencia respiratoria por neumonía, progresión de la falla hepática, hemorragia, deterioro neurológico progresivo, igualmente no más allá de los 3 años.

En nuestros casos el cuadro clínico junto con un aspirado de médula ósea que demostró la presencia de células espumosas (Figura 4) nos hizo sospechar del diagnóstico de enfermedad de Niemann Pick. El aspirado de médula ósea es un procedimiento disponible en nuestro medio, aunque no es diagnóstico nos orienta en el abordaje de manera importante, por lo que nosotros sugerimos su realización. Las células espumosas no son patonogmónicas de la enfermedad pues 


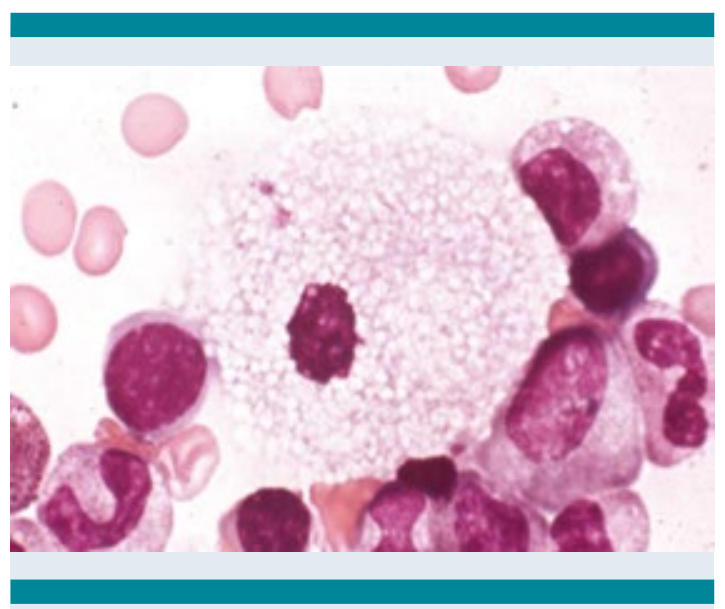

Figura 4. Células espumosas en aspirado de médula ósea en un paciente con enfermedad de Niemann Pick.

se pueden apreciar ocasionalmente en algunos pacientes con talasemia y leucemia mieloide crónica. Al paciente reportado por Salinas Velasco $^{33}$ se le realizó aspirado de médula ósea en 3 ocasiones con resultados negativos y fue la presencia de células espumosas en una biopsia hepática lo que sugirió determinar la actividad de la enzima. ${ }^{33}$

El diagnóstico confirmatorio se realiza al determinar la actividad de la esfingomielinasa ácida en sangre o cultivo de fibroblastos de la piel. ${ }^{34}$ Siendo posible obtener falsos negativos al utilizar un sustrato de esfingomielinasa artificial, 2-N-(hexadecanoilo)-amino-4-nitrofenil fosforilcolina. ${ }^{35}$ El estudio enzimático confirmatorio en nuestros casos se realizó en sangre, estas muestras fueron referidas a otro país ya que en el nuestro no se realiza.

Se ha analizado al biomarcador quitotriosidasa para algunas enfermedades de depósito lisosomal, pero este estudio no es de utilidad para el diagnóstico o seguimiento de la actividad de la enfermedad de Niemann Pick tipo A, a diferencia de lo que se observa en la enfermedad de
Gaucher en donde este biomarcador tiene cierta utilidad. ${ }^{36}$

En ninguno de nuestros pacientes fue posible realizar el estudio molecular, sin embargo, las familias recibieron asesoramiento genético. El diagnóstico prenatal de enfermedad de Niemann Pick tipo A actualmente es factible, a través de la secuenciación del gen SMPD1 en células de biopsia de vellosidades coriales o en amniocitos, así como también es posible determinar la actividad de la esfingomielinasa en las células del líquido amniótico cultivadas a las 17 semanas de gestación. ${ }^{37}$

La enfermedad de Niemann Pick es una enfermedad por depósito lisosomal de baja prevalencia, siendo la tipo A la más frecuente. Con nuestros resultados, aportamos la descripción de una serie de 12 pacientes a la literatura médica. Es importante dar a conocer más casos para establecer las variantes clínicas de la enfermedad, para que con ello más médicos logren reconocer el cuadro clínico de sospecha ante un paciente con una enfermedad neurodegenerativa y hepatoesplenomegalia, posteriormente se realicen estudios complementarios como el aspirado de médula ósea y la revisión de fondo de ojo, tomando en cuenta que en estadios tempranos es posible no encontrar datos sugestivos como células espumosas o la mancha rojo cereza. El diagnóstico definitivo se realiza con la determinación de la actividad de la esfingomielinasa ácida en leucocitos o fibroblastos y el estudio molecular del gen SMPD1. Al no tener un tratamiento específico se debe instaurar una terapia de rehabilitación y de soporte de manera temprana, donde la gastrostomía es el procedimiento que disminuye la frecuencia de neumonías por broncoaspiración. Requiere un manejo multidisciplinario atendiendo los síntomas respiratorios, crisis convulsivas, complicaciones cardiovasculares y alteraciones hematológicas. La terapia de reemplazo enzimático aún se encuentra en etapa experimental. Es 
necesario informar del pronóstico sin alentar el abandono al tratamiento y acompañar a los familiares con apoyo psicológico especializado. La enfermedad de Niemann Pick tipo A es una enfermedad recesiva, por lo que los padres deben recibir asesoramiento genético, pues la probabilidad de tener otro hijo afectado es de $25 \%$, además que en la actualidad existe la posibilidad de poder realizar diagnóstico prenatal.

\section{REFERENCIAS}

1. McGovern MM, Aron A. Natural history of Type A NiemannPick disease: posible endpoints for therapeutic trials. Neurology. 2006;66(2):228-32.

2. Niemann A. Ein unbekanntes Krankheitsbild. Jahrbuch für Kinderheilkunde, Berlin, N F. 1914;79:1-10.

3. Pick L. Der Morbus Gaucher und die ihmähnlichen krankheiten (die lipoid zellige Splenohepatomegalie Typus Niemann und die diabetische Lipoidzellenhypoplasie der Milz). Ergebenisse der Inneren Medizin und KingderheiIkunde, Berlin 1926;29:519-27.

4. Crocker C, Farber S. Niemann-Pick disease: A review of eighteen patients. Medicine, Baltimore, 1958;37:1-95.

5. Meikle PJ, Hopwood JJ, Clague AE, Carey WF. Prevalence of lysosomal storage disorders JAMA. 1999 Jan 20;281(3):249-54

6. Acuña M, Martínez P, Moraga C, He X, Moraga M, Hunter $B$, et al. Epidemiological, clinical and biochemical characterization of the p.(Ala359Asp) SMPD1 variant causing Niemann-Pick disease type B. Eur J Hum Genet. 2015 Feb;24(2):208-13.

7. Simonaro $\mathrm{CM} 1$, Desnick RJ, McGovern MM, Wasserstein $\mathrm{MP}$, Schuchman EH. The demographics and distribution of type B Niemann-Pick disease: novel mutations lead to new genotype/phenotype correlations Am J Hum Genet. 2002 Dec;71(6):1413-9.

8. Graver D. Accurate Differentiation of Neuronopathic and Nonneuronopathic Forms of Niemann-Pick Disease by Evaluation of the Effective Residual Lysosomal Sphingomyelinase Activity in Intact Cells. J Neurochem 1994;63:1060-8.

9. McGovern MM1, Wasserstein MP, Aron A, Desnick RJ, Schuchman EH, Brodie SE. Ocular manifestations of Niemann-Pick disease type B. Ophthalmology. 2004 Jul;111(7):1424-7.

10. McGovern MM. Acid Sphingomyelinase Deficiency. En: Gene Reviews 2006;9:28.

11. 11 Patterson MC, Hendriksz CJ, Walterfang $M$, Sedel $F$, Vanier MT, Wijburg F; NP-C Guidelines Working Group. Recommendations for the diagnosis and management of
Niemann-Pick disease type C: an update. Mol Genet Metab. 2012 Jul;106(3):330-44.

12. Desnick JP, Kim J, He X, Wasserstein MP, Simonaro CM, Schuchman $\mathrm{EH}$. Identification and characterization of eight novel SMPD1 mutations causing types A and B NiemannPick disease. Mol Med. 2010 Jul-Aug;16(7-8):316-21.

13. Schuchman EH. The pathogenesis and treatment of acid sphingomyelinase-deficient Niemann-Pick disease. J Inherit Metab Dis. J Inherit Metab Dis. 2007 Oct;30(5):654-63.

14. Mihaylova V, Hantke J, Sinigerska I, Cherninkova S, Raicheva $M$, Bouwer $S$,et al. Highly variable neural involvement in sphingomyelinase-deficient Niemann-Pick disease caused by an ancestral Gypsy mutation. Brain. 2007 Apr;130(4):1050-61.

15. Irun $\mathrm{P}$, Mallén $\mathrm{M}$, Dominguez $\mathrm{C}$, Rodriguez-Sureda $\mathrm{V}$, Alvarez-Sala LA, Arslan N, Bermejo N, Guerrero C, et al. Identification of seven novel SMPD1 mutations causing Niemann-Pick disease types A and B. Clin Genet. 2013 Oct;84(4):356-61.

16. Bayever E, August CS, Kamani N, Ferreira P, Wenger D, Krivit W. Allogeneic bone marrow transplantation for Niemann-Pick disease (type IA). Bone Marrow Transplant. 1992;10(1):85-6.

17. Daloze P, Delvin EE, Glorieux FH, Corman JL, Bettez P, Toussi T. Replacement therapy for inherited enzyme deficiency: liver orthotopic. Am J Med Genet. 1977;1(2):229-39.

18. Vanier MT, Rousson R, Garcia I, Bailloud G, Juge MC, Revol A, Louisot P. Biochemical studies in Niemann-Pick disease. III. In vitro and in vivo assays of sphingomyelin degradation in cultured skin fibroblasts and amniotic fluid cells for the diagnosis of the various forms of the disease. Clin Genet. 1985 Jan;27(1):20-32.

19. Wasserstein MP, Desnick RJ, Schuchman EH, Hossain S, Wallenstein S, Lamm C, McGovern MM. The natural history of type B Niemann-Pick disease: results from a 10-year longitudinal study. Pediatrics. 2004 Dec;114(6):672-7.

20. Vanier MT. Niemann-Pick disease type C. Orphanet of Rare Disease. 2010;5:1-18.

21. Vanier M T. Niemann-Pick disease. HandbClin Neurol. 2013;113:1717-21.

22. Mohammed MS, Nova Scotia Niemann Pick Disease (Type D) Clinical Study of 20 cases. J Child Neurol. 1998;13:75-78.

23. Macauley SL, Sidman RL, Schuchman EH, Taksir T, Stewart GR. Neuropathology of the acid sphingomyelinase knockout mouse model of Niemann-Pick A disease including structure-function studies associated with cerebellar Purkinje cell degeneration. Exp Neurol. 2008 Dec;214(2):181-92.

24. Aureli M, Prioni S, Mauri L, Loberto N, Casellato R, Ciampa MG, Chigorno V, Prinetti A, Sonnino S. Photoactivable sphingosine as a tool to study membrane microenvironments in cultured cells. J Lipid Res. 2010 Apr;51(4):798-808.

25. Dodge JC, Clarke J, Treleaven CM, Taksir TV, Griffiths DA, Yang W, Fidler JA, Passini MA, Karey KP, Schuchman EH, 
Cheng SH, Shihabuddin LS. Intracerebroventricular infusion of acid sphingomyelinase corrects CNS manifestations in a mouse model of Niemann-Pick A disease. Exp Neurol. 2009 Feb;215(2):349-57.

26. Shihabuddin LS, Numan S, Huff MR, Dodge JC, Clarke J, Macauley SL, Yang W, Taksir TV, Parsons G, Passini MA, Gage FH, Stewart GR. Intracerebral transplantation of adult mouse neural progenitor cells into the Niemann-Pick-A mouse leads to a marked decrease in lysosomal storage pathology. J Neurosci. 2004 Nov 24;24(47):10642-51.

27. Suchi M, Dinur T, Desnick RJ, Gatt S, Pereira L, Gilboa E, Schuchman EH. Retroviral-mediated transfer of the human acid sphingomyelinase cDNA: Correction of metabolic defect in cultured Niemann-Pick disease cells. Proc Natl AcadSci USA. 1992 Apr 15;89(8):3227-31.

28. Dodge JC, Clarke J, Song A, Bu J, Yang W, Taksir TV, et al. Gene transfer of human acid sphingomyelinase corrects neuropathology and motor deficits in a mouse model of Niemann-Pick type A disease. Proc Natl Acad Sci USA. 2005 Dec 6;102(49):17822-7.

29. Schuchman EH. The pathogenesis and treatment of acid sphingomyelinase-deficient Niemann-Pick disease. J Inherit Metab Dis. 2007 Oct;30(5):654-63.

30. Guillemot $N$, Troadec $C$, de Villemeur TB, Clément A, Fauroux B. Lung Disease in niemann-Pick Disease. PediatrPulmonol. 2007 Dec;42(12):1207-14.

31. Hollak CE1, de Sonnaville ES, Cassiman D, Linthorst GE, Groener JE, Morava E, Wevers RA, et al. Acidd sphingomyelinase (Asm) deficiency patients in The Netherlands and Belgium: Disease spectrum and natural course in attenuated patients Mol Genet Metab. 2012 Nov;107(3):526-33.

32. McGovern M, Tilla P, Deckelbaum R, Simpson W. Lipid abnormalities in children with types A and B Niemann Pick Disease. J Pediatr. 2004;145:77-81.

33. Salinas Velasco. Revisión de tema y presentación de caso Enfermedad de Niemann-Pick: A propósito de un caso, Revista chilena de Pediatría, 2009;6(2):718-918.

34. Vanier MT, Rousson R, Garcia I, Bailloud G, Juge MC, Revol A, Louisot P. Biochemical studies in Niemann-Pick disease. III. In vitro and in vivo assays of sphingomyelin degradation in cultured skin fibroblasts and amniotic fluid cells for the diagnosis of the various forms of the disease. Clin Genet. 1985 Jan;27(1):20-32.

35. Harzer K, Rolfs A, Bauer P, Zschiesche M, Mengel E, Backes J, Kustermann-Kuhn B, Bruchelt G, van Diggelen OP, Mayrhofer $\mathrm{H}$, Krägeloh-Mann I. Niemann-Pick disease type A and $B$ are clinically but also enzymatically heterogeneous: pitfall in the laboratory diagnosis of sphingomyelinase deficiency associated with the mutation Q292 K. Neuropediatrics. 2003 Dec;34(6):301-6.

36. Ries $M$, Schaefer E, Lührs $T$, Mani L, Kuhn J, Vanier MT, Krummenauer F, Gal A, Beck M, Mengel E. Critical assessment of chitotriosidase analysis in the rational laboratory diagnosis of children with Gaucher disease and Niemann-Pick disease type A/B and C. J Inherit Metab Dis. 2006 Oct;29(5):647-52.

37. Higami S, Omura K, Nishizawa K, Yamashita T, Tada K. Prenatal diagnosis and fetal pathology of Niemann-Pick disease. Tohoku J ExpMed. 1978;125(1):11-7. 